\title{
Discrimination of third-order Markov constraints within visual displays*
}

\author{
IRWIN POLLACK \\ Mental Health Research Institute, University of Michigan, Ann Arbor, Michigan 48104
}

\begin{abstract}
Discrimination thresholds were measured for third-order Markov constraints within visual displays. The method permits the cancellation of adjacent second-order differences and of first-order differences. Excellent discrimination of third-order constraints is obtained despite the fact that the average conditional repetition probability and the average run length were invariant. The need to distinguish between local short-term and average long-term analyses for the visual system is briefly discussed.
\end{abstract}

For several years, I have been attempting to explore methods which permit the "perceptualization" of probabilistic information. Consider the prototype, Humphreys's (1939) guessing experiment where, on a given trial, the $S$ must decide which of two lights will occur. Such experiments proceed at the rate of about 1 trial/ $10 \mathrm{sec}$. The investigator derives a trial-by-trial record of the S's responses as a function of the probabilistic structure of the sequence which drives the lights. In order to sample the S's sensitivity to such sequences, one can improve the efficiency of the experiment by asking the $S$ to respond to the lights in a choice reaction time test (Bertleson, 1961, 1963; Kornblum, 1968). Such experiments might proceed at the rate of $1 \mathrm{trial} / \mathrm{sec}-\mathrm{a} 10^{1}$-fold improvement. A further improvement is achieved by the rapid presentation of sequences of individual items-to rates of 4 items/sec-and asking the 0 to reproduce the sequence at his own speed (Restle, 1970; Preusser, Garner, \& Gottwald, 1970).

If we drop the requirement that the $S$ respond to each item of the sequence, the same binary-coded materials can be presented at the rate of $10^{4}$ samples $/ \mathrm{sec}$ for auditory signals (Pollack, 1971b)-a $10^{5}$-fold improvement over the original experiment-or several thousand samples in a short visual flash. The "information speed-up" essentially converts the initial "cognitive-intellectual" task into a "perceptual" task. Such conversion takes advantage of the built-in machinery available for perception, e.g., spectral frequency analysis (cf. Cornsweet, 1971; Plomp \& Smoorenburg, 1970) or binocular disparity (Julesz, 1960).

\footnotetext{
*The research was supported in part by Grant GBX14036X from the National Science Foundation. The author wishes to acknowledge the assistance of Nancy Mandell for supervision of the experimental tests, Louis Wojnaroski for the PDP-9 computer program, and Robert Shea for processing the experimental results.
}

The present paper examines the sensitivity to third-order statistical constraints encoded within visual displays. We ask whether such constraints can be discriminated in the absence of nonadjacent second-order constraints and in the absence of first-order constraints.

\section{APPROACH}

\section{Definition of the Order of the Constraint}

Consider a sequence of binary elements of $1 \mathrm{~s}$ and $0 \mathrm{~s}$. We can vary the first-order probabilities of the sequence by varying the probability of State $1, P(1)$. If the two states are encoded in the form of brightness, e.g., where $1=$ dot and $0=$ no dot, such binary sequences can be translated into sequences of dots and of nondots. A single long linear sequence can be broken and laid down as smaller adjacent linear subsequences upon a plane. For example, a single linear sequence of 100 items may be broken into 10 sequences and laid down in a 10 by 10 matrix. Variations in $P(1)$ will be reflected in brightness differences over the display. Indeed, Green, Wolf, and White (1959) and Julesz (1962) have obtained threshold differences for displays of this type.

Consider now pairs of elements. Two forms of transition are defined. A repetition ( $R$ ) is defined if 1 follows 1 or if 0 follows 0 ; an alternation (A) is defined if 1 follows 0 or if 0 follows 1 . If $P(R)=P(1 \mid 1)=P(0 \mid 0)$, then $P(1)=P(0)$, and variation in $P(1 \mid 1)$ defines the entire second-order transitional matrix without differences in first-order probabilities. Sequences in which $P(1 \mid 1)$ is varied can be translated into sequences of dots and spaces. Such sequences will differ in terms of their texture, where high repetition probabilities lead to "course" textures (dots following dots and spaces following spaces) and low repetition probabilities lead to "even" or "lacey" textures (dots following spaces and spaces following dots). Indeed, Harcum (1967), Julesz (1962), Pickett (1964, 1967), and Pollack (1971a) have obtained responses for displays of this type.

Consider now pairs of transitions. A repetition can follow a repetition to produce sequences such as 111 or 000 or an alternation can follow an alternation to produce such sequences as 101 or 010 . If $P(R \mid R)=P(A \mid A)$, then $P(R)=P(A)$, and third-order differences can be obtained in the absence of immediately adjacent second-order differences and in the absence of first-order differences. Such sequences will differ in terms of their mixture of textures. High transitional probabilities lead to mixtures of course and even textures. Low transitional probabilities lead to even mixtures of textures. 
An interesting feature of controlled third-order sequences, where $P(R)=P(A)$, is that the average run length is invariant as $P(R \mid R)$ and $P(A \mid A)$ are changed. Thus, if modifications in $P(R \mid R)$ and $P(A \mid A)$ can be discriminated, we cannot point to average run length as the basis of discrimination. Finally, it is noted that the method outlined for developing third-order differences with binary-encoded sequences may also introduce nonadjacent second-order differences. For example, at high $P(R \mid R)$ and $P(A \mid A)$ levels, sequences such as $11111 \ldots$ and $10101 \ldots$ are highly probable. These sequences also reflect a high second-order probability among elements two units removed. Julesz (1962) has shown that when the number of symbols of the sequence-the number of brightness levels in a visual display-meets a defined criterion, third-order differences can still be detected in the absence of any second-order differences.

\section{Definition of the Dimensionality of the Constraints}

The previous section defined the order of the constraint in terms of a linear sequence of binary elements. Displays based upon a single linear sequence or upon a concatenation of linear sequences are defined as one-dimensional displays.

Displays can also be defined in terms of two-dimensional constraints. A two-dimensional constraint in the $x$ and $y$ coordinates may be based upon combinations of three (Julesz, 1962) or four (Pollack, 1971a, c) display elements. Again, let us assume that $1=$ dot and $0=$ no dot. In the context of two-dimensional constraints over four elements, an even parity sum, $\Sigma_{e}$, requires that 0,2 , or 4 dots appear within defined 2 by 2 cells of display elements, and an odd parity sum requires that 1 or 3 dots appear within the cell. If three of the elements have previously been selected, the parity sum will determine the fourth element of the cell. For example, if three previously selected elements were no-dots, an even parity sum would require that the fourth element would be a no-dot $(0+0=$ even), and an odd parity sum would require that the fourth element would be a dot $(0+1=$ odd $)$. Correspondingly, had the three previously selected elements been dots, an even parity sum requires that the fourth element be a dot $(3+1=$ even $)$, and an odd parity sum requires that the fourth element be a nondot ( 3 $+0=$ odd).

Variation in the probability of an even parity sum, $P\left(\Sigma_{e}\right)$, manipulates the second-order, two-dimensional constraints in the same manner as variation in the repetition probability, $P(R)$, manipulates second-order, one-dimensional constraints. Variation in the transition probability. $P\left(\Sigma_{e} \mid \Sigma_{e}\right)$, manipulates third-order, two-dimensional constraints.

\section{Task}

In a given experimental session, 0 was presented displays either with one-dimensional constraints or with two-dimensional constraints. A given observation consisted of the simultaneous presentation of four matrices of dots and no-dots, each upon an invisible 32 by 32 grid. The interdot spacing was $1.17 \mathrm{~mm}$; the dot width was about $0.8 \mathrm{~mm}$; the displays were painted upon the $8 \times 8 \mathrm{~cm}$ display surface of a Tektronix 602 display scope equipped with a fast P-15 phosphor by a PDP-9 computer. The duration of the display was controlled by successively repainting the display 8,32 , or 128 times to achieve durations of $0.15,0.6$, or $2.4 \mathrm{sec}$, respectively. O varied his seating distance for maximum comfort with natural binocular viewing.

Three of the four matrices obeyed one transitional probability $-P(R \mid R)_{r}$ in the case of one-dimensional constraints, and $P\left(\Sigma_{e} \mid \Sigma_{e}\right)_{r}$ in the case of two-dimensional constraints. One of the four matrices obeyed another transitional probability-P(R, $\mid R)_{y}$ in the case of one-dimensional constraints, and $P\left(\Sigma_{\mathbf{e}} \mid \Sigma_{\mathbf{e}}\right)_{\mathrm{V}}$ in the case of two-dimensional constraints. The position of the odd matrix was varied at random. O's task was to identify. by pressing one of four buttons, whether the northwest, the nor theast, the southwest, or the southeast matrix was the "odd" matrix. Immediate feedback was given. After a correct response, the magnitude of the difference between the transitional probabilities was reduced by modifying the variable transitional probability. After an incorrect response, the magnitude of the difference was increased. The variable transition repetition probability was manipulated by an adaptive stimulus programming procedure (Taylor \& Creelman, 1967) to converge upon 50\% correct responses in the four-alternative forced-choice test.

Each threshold represents the geometric mean of $17 \mathrm{Os}$ contributing two thresholds. With 99 experimental conditions, about $3.4 \times 10^{3}$ thresholds are represented.

Successive constraints were plotted vertically. For example, the string . . ARRRA ... headed by a nondot was translated to a vertical string of . . . no dot, four dots, no dot . ... Successive vertical positions also marked the sequence of parity sums with two-dimensional constraints.

\section{RESULTS}

Figure 1 presents one-dimensional transition repetition thresholds. The coordinates are those of the normal probability grid. The 45 -deg reference line represents infinite discriminability. The shape of the points represents the three durations employed. A straight line of slope 1.0 signifies that a constant standard-score difference in transition repetition probabilities is required at threshold. Slight, but consistent, differences were obtained as a function of display duration. In terms of normalized difference scores, a vertical threshold difference of 0.14 units is required, when averaged over display duration, for the detection of increments in transition repetition probability above the reference level (points plotted above the reference line), and a vertical threshold difference of 0.16 normalized units is required for the detection of decrements in transition repetition probability below the reference level. The perpendicular distance to the line, $d^{\prime}$ is the vertical normalized distance multiplied by $\sqrt{2}$ (Green \& Swets, 1966).

The implication of the method of plotting Fig. 1, and later of Fig. 2, is opposite to that stemming from typical experiments in signal detection. There, the normalized coordinates typically describe a receiver operating characteristic, or changes in the detection performance obtained for a fixed signal level. The larger the deviation from the reference axis, the better the performance for the fixed signal level. Here, the coordinates describe a signal operating characteristic, or changes in the signal conditions required for a fixed performance level. The larger the deviation from the reference axis. the poorer the performance, since larger differences in signal level are required for a constant performance level.

Figure 2 presents two-dimensional transition repetition thresholds. The organization of Fig. 2 is parallel to that of Fig. 1. The picture is more complex than that of Fig. 1 because of the more powerful role of display duration and because of the nonunity slope. In the vicinity of a reference transition repetition probability of 0.50 . a vertical difference of about .25 
Fig. 1. Third-order one-dimensional constraint thresholds for visual displays. The coordinates are those of the normal probability grid. The abscissa is the reference conditional transition probability for a repetition following itself or for an alternation following itself. Increment thresholds are represented above the reference diagonal; decrement thresholds are represented below the reference diagonal. Display duration is coded by the shape of the points.

Fig. 2. Third-order two-dimensional constraint thresholds for visual displays. As Fig. 1, except that the coordinates describe the conditional transition probability of an even parity sum following itself or of an odd parity sum follnwino itceif
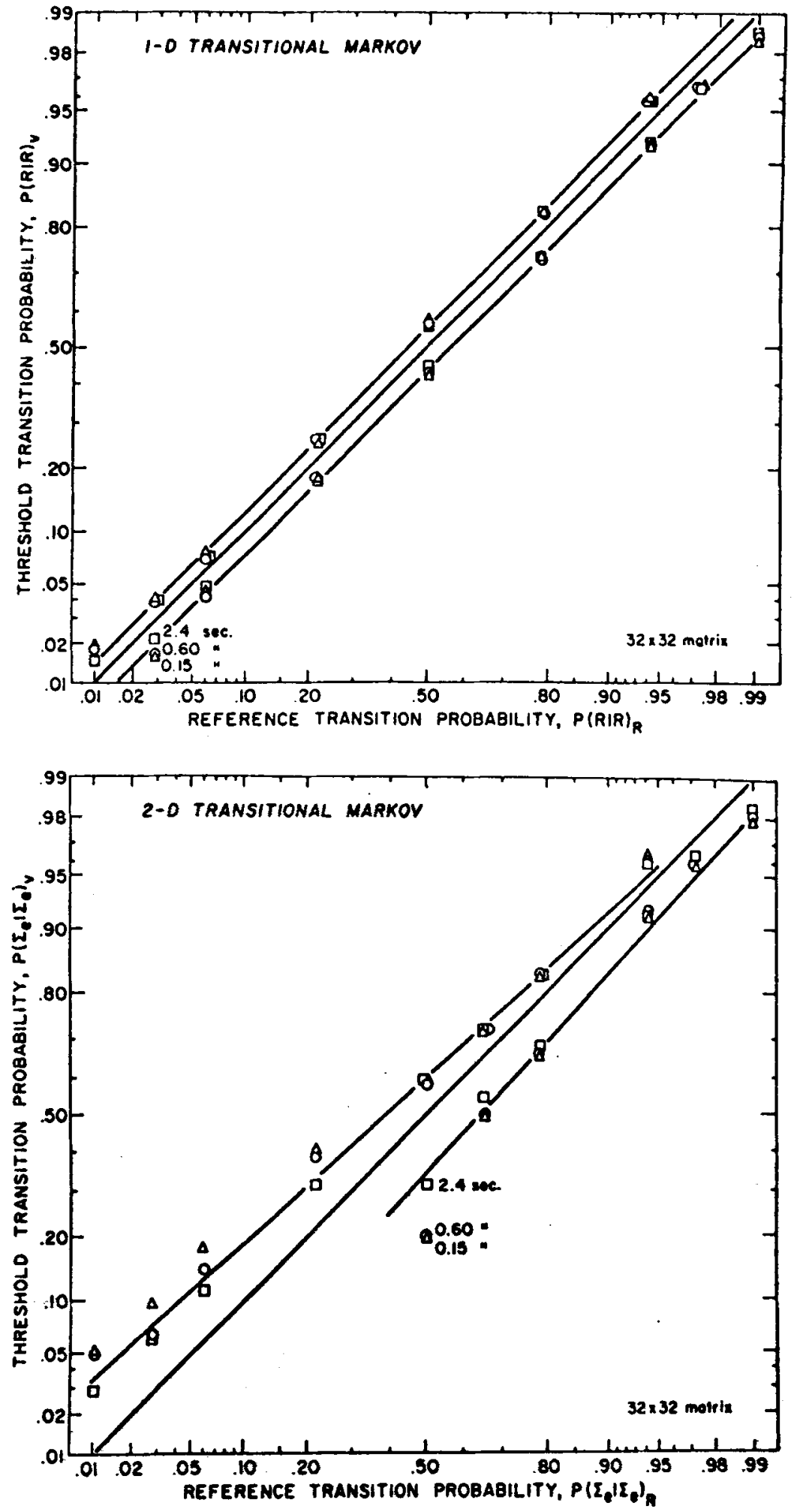

normalized units is required for the detection of increments in transition repetition probability, and .40 normalized units is required for the detection of decrements, when averaged over display duration. Nearly twice the normalized difference is required for the two-dimensional constraints, relative to that required for the one-dimensional constraints.

Not shown on the grid of Figs. 1 and 2 are thresholds for extreme reference transitional probabilities. These are tabulated in Table 1. Neglecting the fact that a 
Table 1

Thresholds for Extreme Transitional Reference Probabilities

\begin{tabular}{ccccc}
\hline \multirow{2}{*}{$\begin{array}{c}\text { Reference } \\
\text { Probability }\end{array}$} & $\begin{array}{c}\text { Dimen- } \\
\text { sionality }\end{array}$ & .15 & 0.6 & 2.4 \\
\cline { 3 - 5 } .0001 & 1-D & .0037 & .0021 & .0016 \\
& 2-D & .0147 & .0125 & .0093 \\
.9999 & 1-D & .9992 & .9992 & .9993 \\
& 2-D & .9978 & .9979 & .0082 \\
\hline
\end{tabular}

32 by 32 grid provides only 1,024 elements, we might assign to the reference probabilities of .0001 and $.9999 \mathrm{a}$ normalized score of 3.73 units. The median threshold differences are then $0.87,1.49,0.58$, and 0.87 normalized units, respectively, when averaged over display duration for the conditions of Table 1. The estimates are out of line with those of the less extreme reference conditions.

We can attempt to finesse the problem of specifying the extreme reference probabilities by deriving an implied set of reference probabilities, based on the assumption that the normalized difference scores remain invariant. For the reference probability $\mathrm{P}=.9999$, the implied levels are $\mathrm{P}=.9995$ and $\mathrm{P}=.9994$ for $1-\mathrm{D}$ and 2.D constraints, respectively. For the reference probability $\mathrm{P}=.0001$, the implied levels are $\mathrm{P}=.0013$ and $P=.0064$ for 1-D and 2-D constraints, respectively. To the extent that these implied levels are considered unreasonable for a matrix of 1,024 elements, the analysis suggests that larger normalized differences are required at lower extreme probability levels than at higher extreme probability levels.

\section{DISCUSSION}

Threshold sensitivities for third-order Markov constraints closely parallel those for second-order Markov constraints (Pollack, 1972). In the latter case, 1-D thresholds of about 0.16 normalized units and 2-D thresholds of $.32-39$ normalized units were obtained at long durations, and 1-D thresholds were typically less sensitive to display duration. In the present case, 1-D thresholds of about 0.16 normalized units and 2-D thresholds of about .25-.40 normalized units were obtained. Thus, there was apparently little performance penalty for the extra order of statistical constraint in the present tests. This result suggests that third-order constraints were not perceived by a concatenation of nonadjacent second-order constraints.

A number of investigators have proposed that people are unusually sensitive to the run properties of auditory and visual stimuli (Preusser, Garner, \& Gottwald, 1970; Restle, 1970; Vitz, 1968). Indeed, the Os in the present study may have been responding to run properties in the displays, but we can rule out the possibility that they were responding to the average run length, ARL, of the displays. The ARL is dependent upon the average second-order conditional repetition probability. which remained fixed at 0.5 , and $A R L=2$. Consider three extreme examples. When $P(R \mid R)=P(A \mid A) \sim 0$, then we have sequences of the forms ARARAR ..., which translated into states $0110011 \ldots$ with $A R L=2$; when $\mathrm{P}(\mathrm{R} \mid \mathrm{R})=\mathrm{P}(\mathrm{A} \mid \mathrm{A})=0.50$, we have random sequences of $A$ and $R$ in which strings of As balance strings of $R s$ with a resultant $A R L=2$; and when $P(R \mid R)=P(A \mid A)$ $\sim 1.0$, we have very long sequences of Rs and very long sequences of As: A string of Rs of length $n$ would yield 1 sequence of run length of $n$; a string of As of length $n$ would yield $n$ sequences of run length of 1 ; the ARL would be $(1 \cdot n)+(n \cdot 1) /(n+1)$ and, at large $n, A R L=$ 2.

While the average run length is independent of $P(R \mid R)$, local runs are not. At high $P(R \mid R)$ levels, long runs are obtained; at high $\mathrm{P}(\mathrm{A} \mid \mathrm{A})$ levels, long alternating strings are obtained. The distinction between local and average properties is important and is often overlooked. For example, the auditory system has long been considered an excellent model for Fourier frequency analysis. A pulse train of random polarity has a wide long-term spectrum approaching wide-band noise. Such pulse trains sound noisy, but at infrequent intervals, individual "chirps" can be heard (Cramer \& Licklider, 1957). Presumably, local runs of identical polarity or of alternating polarity can be extracted by a running or short-term analysis of the auditory signal, leading to a local tonality. Phenomenal report and the present results suggest that the visual system also responds in terms of local or short-term rather than average or long-term run-length features.

\section{REFERENCES}

Bertelson, P. Sequential redundancy and speed in a serial two-choice responding task. Quarterly Journal of Experimental Psychology, 1961, 13, 90-102.

Bertelson, P. S-R relationships and reaction times to new versus repeated signals in a serial task. Journal of Experimental Psychology, 1963, 65, 478-484.

Cornsweet, T. Visual perception. London: Academic Press, 1970.

Cramer, E. M., \& Licklider, J. C. R. Pitch of a train of pulses of random polarity. Journal of the Acoustical Society of America, 1957, 29, 780(A).

Green, B. F., Jr., Wolf, A. K., \& White, B. W. The detection of statistically defined patterns in a matrix of dots. American Journal of Psychology, 1959, 72, 503-520.

Green, D. M., \& Swets, J. Signal detection theory and psychophysics. London: Wiley, 1966.

Harcum, E. R. Visual detection and recognition of targets with various dependency contrasts in microstructure. Journal of Experimental Psychology, 1967, 73, 155-159.

Humphreys, L. G. Acquisition and extinction of verbal expectations in a situation analogous to conditioning. Journal of Experimental Psychology, 1939. 25, 294-301.

Julesz, B. Binocular depth perception of computer-generated patterns. Bell Systems Technical Journal. 1960, 39. 1125-1162.

Julesz. B. Visual pattern discrimination. Institute of Radio Engineers Transactions on Information Theory. 1962. IT-8. 84-92. 
Kornblum, S. Serial-choice reaction-time: Inadequacies of the information hypothesis. Science, 1968, 159, 432-434.

Pickett, R. M. The perception of a visual texture. Journal of Experimental Psychology, 1964, 68, 13-20.

Pickett, R. M. Response latency in a pattern perception situation. In A. F. Sanders (Ed.), Attention and performance. Amsterdam: North Holland, 1967.

Plomp, R., \& Smoorenburg, G. F. Frequency analysis and periodicity detection in hearing. Leiden: Sijthoff, 1970.

Pollack. I. Perception of two-dimensional Markov constraints within visual displays. Perception \& Psychophysics, 1971a, 9. $461-464$.

Pollack, I. Depth of sequential auditory information processing: III. Journal of the Acoustical Society of America, 1971b, 50, 549-554.

Pollack, I. Detection of one-, two-, and three-dimensional Markov constraints in visual displays. Acta Psychologica, 1971c, 35, 219-232
Pollack, I. Visual discrimination thresholds for one- and two-dimensional Markov spatial constraints. Perception \& Psychophysics, 1972, 12, 161-167.

Preusser, D., Garner, W. R.. \& Gottwald, R. L. Perceptual organization of two-element temporal patterns as a function of their component one-element patterns. American Journal of Psychology, 1970, 83, 151-170.

Restle, F. Theory of serial pattern learning: Structural trees. Psychological Review, 1970, 77, 481-495.

Taylor, M. M.. \& Creelman, C. D. PEST: Effficient estimates on probability functions. Journal of the Acoustical Society of America, 1967, 41, 782-787.

Vitz, P. C. Information, run structure and binary complexity. Perception \& Psychophysics, 1968, 3, 275-280.

(Received for publication September 29, 1972; revision received November 15,1972 .) 